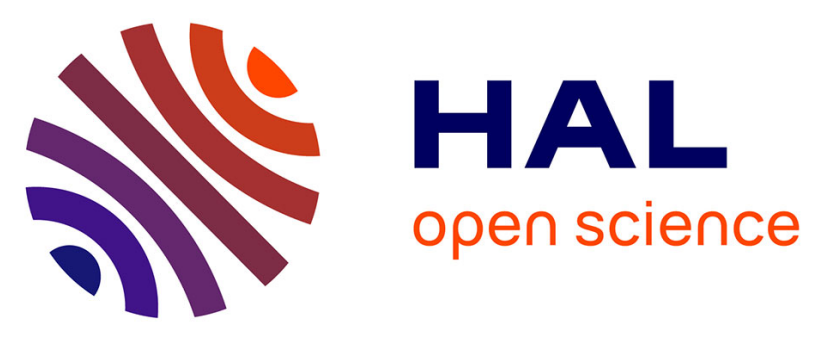

\title{
Preparation of polymer supported phosphine ligands by metal catalyzed living radical copolymerization and their application to hydroformylation catalysis
}

Andrés Fernando Cardozo Perez, Eric Manoury, Carine Julcour-Lebigue, Jean-François Blanco, Henri Delmas, Florence Gayet, Rinaldo Poli

\section{To cite this version:}

Andrés Fernando Cardozo Perez, Eric Manoury, Carine Julcour-Lebigue, Jean-François Blanco, Henri Delmas, et al.. Preparation of polymer supported phosphine ligands by metal catalyzed living radical copolymerization and their application to hydroformylation catalysis. ChemCatChem, 2013, Homogeneous Catalysis, 5 (5), pp.1161-1169. 10.1002/cctc.201200446 . hal-00993527

\section{HAL Id: hal-00993527 \\ https://hal.science/hal-00993527}

Submitted on 29 Jul 2021

HAL is a multi-disciplinary open access archive for the deposit and dissemination of scientific research documents, whether they are published or not. The documents may come from teaching and research institutions in France or abroad, or from public or private research centers.
L'archive ouverte pluridisciplinaire HAL, est destinée au dépôt et à la diffusion de documents scientifiques de niveau recherche, publiés ou non, émanant des établissements d'enseignement et de recherche français ou étrangers, des laboratoires publics ou privés. 


\title{
Preparation of Polymer Supported Phosphine Ligands by Metal Catalyzed Living Radical Copolymerization and Their Application to Hydroformylation Catalysis
}

\author{
Andrés F. Cardozo[a],[b],[c], Eric Manoury ${ }^{[a],[c]}$, Carine Julcour ${ }^{[b],[c]}$, Jean-François \\ Blanco $^{[b],[c]}$, Henri Delmas ${ }^{[b],[c]}$, Florence Gayet, ${ }^{[a],[c]}$ Rinaldo Poli ${ }^{*[a],[c],[d]}$
}

A series of well defined polystyrene supported tertiary phosphine ligands were prepared by copper catalyzed atom transfer radical polymerization (ATRP), involving direct copolymerization of styrene and 4-styryldiphenylphosphine (SDPP). Copolymerization of the two monomers at different molar ratios showed a decreasing level of control as the SDPP molar fraction ( $\left.f_{S D P P}\right)$ increased, with a satisfactory level of control being achieved for $\mathrm{f}_{\mathrm{SDPP}} \leq 0.25$ (constant

\section{Introduction}

The ability to construct functionalized polymers with precise architecture, topology, composition, molecular weight, mole fraction and location of specific functional groups leads to an essentially limitless number of advanced materials with specific functions. ${ }^{[1]}$ In this area, giant steps have been made in recent times by the development of controlled radical polymerization techniques, because they combine the precision in macromolecular synthesis of living/controlled polymerization and the typical advantages of radical polymerization, namely the large choice of polymerizable monomers and the tolerance to impurities. ${ }^{[2-6]}$

Among the functionalities that can be attached to a polymer backbone, phosphines have so far received little attention with respect to the controlled radical polymerization approach. On the other hand, phosphine functionalized polymers have been prepared and studied for quite some time by the catalytic chemists' community. ${ }^{[7-8]}$ For instance, polymer-supported rhodium complexes were first described by Manassen ${ }^{[9]}$ and then developed in the 70's by Grubbs, ${ }^{[10-11]}$ Čapka ${ }^{[12]}$ and Pittman ${ }^{[13]}$ as hydrogenation, hydrosilylation and hydroformylation catalysts. Since then, many polymer-supported phosphine (PSP) ligands have been reported in the literature. ${ }^{[7,14-16]}$ Many strategies have been used to access PSP ligands, mostly leading to materials with anchored triphenylphosphine. These can be divided into two classes, the first one involving chemical modification of a preexisting phosphine-free polymer and the second one involving the introduction of phosphine-containing monomers during the macromolecular synthesis. An example of the first strategy is treatment of bromo functionalized polystyrene resins with butyllithium and subsequent chlorodiphenylphosphine addition. ${ }^{[17]}$ Merrifield resins, containing chloro- or bromomethyl anchoring functions can be treated with lithium or potassium diphenylphos- concentration of growing "living chains", linear $\mathrm{Mn}$ growth with conversion and low dispersity). Copper-free polymers with different chain lengths were prepared and tested as polymeric ligands in the Rh-catalyzed hydroformylation of 1-octene. The polymeric ligands yielded higher linear/branched selectivity and lower activity relative to $\mathrm{PPh}_{3}$ at the same P/Rh ratio. The selectivity increased slightly as a function of the polymer chain length.

phide to obtain supported benzyldiphenylphosphine ligands. ${ }^{[14,18]}$ By the second strategy, 4-diphenylphosphinostyrene (or styryldiphenylphosphine, SDPP) can be polymerized or copolymerized together with regular styrene $(S)$ by anionic or free radical methods. Free radical SDPP/S copolymerization in the absence or presence of additional crosslinking comonomers (for instance p-divinylbenzene or 1,4-bis(4-vinylphenoxy)-butane) has given access to soluble linear or insoluble cross-linked materials, having however broad molecular weight distributions given the uncontrolled nature of the polymerization. ${ }^{[19-20]}$ Anionic living polymerrization of SDPP has provided homopolymers with narrow MW distribution, subsequently extended into diblock copolymer structures by polymerization of regular styrene. ${ }^{[21]}$ An alternative way that has been described is radical polymerization of SDPP initiated by radical functions that are generated by $\gamma$-radiation of a polypropylene support, resulting in the build-up of short surfacegrafted SDPP homopolymer chains. ${ }^{[22]}$ More sophisticated systems, containing bulky phosphines ${ }^{[23]}$ and phosphites, ${ }^{[24]}$ have also been made, usually by the polymer chemical functionalization strategy.

\footnotetext{
[a] Mr. A. Cardozo Dr. E. Manoury, Dr. F. Gayet, Prof. R. Poli CNRS, LCC (Laboratoire de Chimie de Coordination) 205 route de Narbonne BP 44099, F-31077 Toulouse Cedex 4, France Fax: (+)33-561553003 E-mail: rinaldo.poli@lcc-toulouse.fr

[b] Dr. C. Julcour, Dr. J.-F. Blanco, Prof. H. Delmas CNRS, LGC (Laboratoire de Génie Chimique) 4 Allée Emile Monso BP 84234, 31030 Toulouse Cedex 4, France

[c] Université de Toulouse, UPS, INPT, F-31077 Toulouse Cedex 4 France

[d] Institut Universitaire de France, 103, bd Saint-Michel, 75005 Paris, France
} 
The above methods generally yield materials where the location and relative amount of phosphine functions cannot be perfectly controlled. We are aware of only one precedent for the incorporation of a phosphine functionalized monomer into a copolymer by controlled radical polymerization. However, this involved the simultaneous incorporation of the phosphine functionalized monomer (SDPP), a halogen-terminated polyacrylate macroinitiator, and a cross-linker (ethylene glycol dimethyl dimethacrylate), by a ruthenium-catalyzed atom transfer approach, to yield core cross-linked and phosphine-functionalized core-shell copolymers. ${ }^{[25-26]}$ In this contribution, we wish to report the synthesis of simpler linear triphenylphosphine containing polystyrene chains by controlled SDPP/S radical copolymerization. In particular, we wished to establish whether an equally good level of control in terms of $M_{n}$ and molecular weight distribution (as measured by the dispersity index, $\oslash=M_{w} / M_{n}$ ) is obtained for different levels of SDPP incorporation, or how these parameters would depend on it, and whether the controlled polymer microstructure would have an effect on the ligand performance in a benchmark catalytic application, the Rhcatalyzed hydroformylation of linear $\alpha$-olefins (represented for this study by 1-octene).

\section{Results and Discussion}

\section{(a) Polymer synthesis and characterization}

As stated in the introduction, the controlled incorporation of SDPP in a copolymer structure by atom transfer radical polymerization (ATRP) has already been reported with use of complex $\mathrm{RuCl}_{2}\left(\mathrm{PPh}_{3}\right)_{3}$ as the atom transfer catalyst. However, since the functionalized monomer can bind the ruthenium catalyst just as well as $\mathrm{PPh}_{3}$, the polymer that was obtained contained the $\mathrm{Ru}$ catalyst coordinated by the polymer-anchored phosphine functions and entrapped within the polymer star core. Although a procedure to completely remove the metal from the polymer exists, we wished to simplify the procedure and used instead the $\mathrm{CuBr} / \mathrm{Me}_{6}$ TREN system as ATRP catalyst. This catalytic system yields a fast and well controlled polymerization of styrene resulting in polymers with target molecular weights and low dispersities. ${ }^{[27]}$ Moreover, the chelating power of the tetradentate $\mathrm{Me}_{6}$ TREN should ensure efficient sequestering of the copper ion, inhibiting any copper coordination by the phosphine functions present in the monomer and polymer. A potential problem under ATRP conditions is attack of the alkyl halide chain end (a benzylic halide for styrene polymerization) by the tertiary phosphine function of the monomer or polymer, inducing formation of quaternary phosphonium salts (see Scheme 1). A second potential problem is dehydrobromination of the halogenated chain end, leading to an unsaturated chain end and $\mathrm{HBr}$. Although this phenomenon has never been reported, to the best of our knowledge, for the ATRP of styrene under the same conditions, it may be favored in our case by the presence of the phosphine functions. These reactions are highly undesirable since they would lead to consumption of the halogen chain ends and formation of dead chains and networks. It is therefore necessary to probe the possible presence and extent of these reactions under the polymerization conditions.

Previous work has shown that the halogenated chain end of polystyrene and polyacrylates prepared by ATRP may indeed be transformed into phosphonium salts upon addition of phosphines, the reaction becoming faster upon increasing the temperature and the solvent polarity (i.e. THF $<$ DMSO $<$ acetonitrile). ${ }^{[28]} \mathrm{We}$ therefore decided to test for the presence of this reaction in toluene, which is the solvent chosen for our polymerization reaction. The halide chain end was emulated by 1phenylethylbromide and the reactive phosphine function was emulated by $\mathrm{PPh}_{3}$. In order to model the polymerization conditions as closely as possible, a large $\mathrm{PPh}_{3} /$ alkyl bromide ratio (12.5) was used. The same copper catalyst system as in the ATRP process was also added to the reaction mixture and the mixture was heated to $100^{\circ} \mathrm{C}$ for several hours with continuous ${ }^{1} \mathrm{H}$ and ${ }^{31} \mathrm{P}$ NMR monitoring. Up to $26 \mathrm{~h}$ (covering the time range used for the polymerization process), no signals other than those of the reactants were observable. In particular, the $\mathrm{PPh}_{3}$ singlet at $\delta-4.89$ remained the only observable resonance in the ${ }^{31} \mathrm{P}$ spectrum. This can be compared with a $20 \%$ conversion after 24 $\mathrm{h}$ when using the same reagents at $80^{\circ} \mathrm{C}$ in acetonitrile. ${ }^{[28]}$ Therefore, the nucleophilic substitution side-reaction should not take place under our ATRP conditions. The possible presence of dehydrobromination was probed by ${ }^{1} \mathrm{H}$ NMR spectroscopy on the prepared polymer samples (vide infra).

Copolymers made from comonomer mixtures with $\mathrm{f}_{\mathrm{SPDD}}$ of 0 , $0.15, \quad 0.25, \quad 0.5$ and 1 were prepared by ATRP with $\mathrm{CuBr} / \mathrm{Me}_{6}$ TREN as catalyst in order to study the incorporation behavior of the phosphine monomer. The choice of ethyl bromoisobutyrate $(\mathrm{EBiB})$ as initiator was made on the basis of its faster activation relative to the oligomeric secondary radical species generated after styrene or SDPP addition, thus ensuring fast initiation. ${ }^{[29]} \mathrm{A}$ small amount $(10 \%)$ of $\mathrm{CuBr}_{2}$ deactivator was added to the reagent mixture to prevent a slower deactivation rate relative to the propagation rate at the beginning of the reaction. The other conditions were inspired by the literature report on styrene homopolymerization (see experimental section). ${ }^{[27]}$ It should also be mentioned that the SDPP/S reactivity ratios for radical copolymerization have been determined under free radical polymerization conditions $\left(r_{S}=0.52, r_{S D P P}=1.43\right),{ }^{[19]}$ indicating that SDPP should be incorporated into the macromolecular chain slightly faster than styrene. As generally admitted, these parameters should not change when transposed to an ATRP process. ${ }^{[30-31]}$ meaning that the SDPP/S copolymerization should yield a slight compositional gradient copolymer with a higher inclusion of SDPP at the beginning of the polymerization and lower at the end, relative to the ratio in the comonomer initial feed. The results obtained are shown in Table 1.

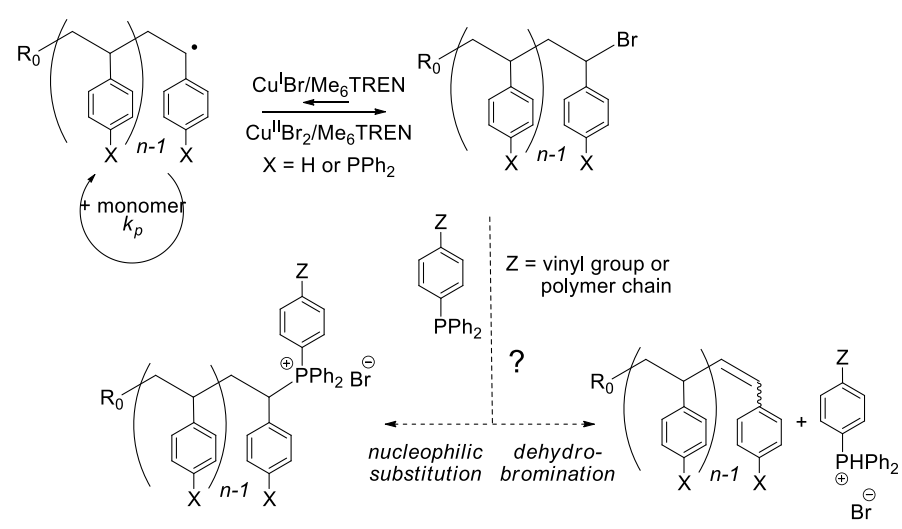

Scheme 1. Atom transfer process and potential nucleophilic attack by phosphine for the dormant chain end of PS-Co-PSDPP made by ATRP. 
After purification, all polymers were white indicating removal of the $\mathrm{Cu}$ salts. This demonstrates that the copper ion has greater affinity for the chelating $\mathrm{Me}_{6}$ TREN ligand than for the polymer supported phosphine. The possibility that copper remains trapped as a less colored phosphine complex in the reduced $\mathrm{Cu}^{\prime}$ state in the polymer was checked by a UV-visible study (see SI); the fraction of residual copper remaining in the polymer has been estimated as ca. $1.5 \%$ of the amount used for the polymer synthesis. This result is at variance with the $\mathrm{RuCl}_{2}\left(\mathrm{PPh}_{3}\right)_{3^{-}}$ catalyzed ATRP leading to SDPP-containing polymer nanogels, where the catalyst remains trapped into the polymer. ${ }^{[25]}$ The monomer units incorporated in the polymer backbone exhibit a different ${ }^{31} \mathrm{P}$ NMR resonance ( $\delta \mathrm{ca}$. -5.8 , broader) from that of the free monomer ( $\delta-5.39$, sharper), see Figure 1. In a few cases, traces of oxide were found in the final product, nevertheless they accounted for no more than $1 \%$ of the sample or recovered polymer in each case. No other signals were detected on the ${ }^{31} \mathrm{P}$ NMR spectra.

The SDPP monomer conversion could be determined by deconvolution of the ${ }^{31} \mathrm{P}$ NMR signal. The advantage of using ${ }^{31} \mathrm{P}$ NMR is the simplicity of the spectrum and the fact that the two resonances of interest are well separated from those of potential impurities (notably traces of phosphine oxide). The SDPP homopolymerization ( $f_{\text {SDPP }}=1$ ) was fast and uncontrolled, reaching $86 \%$ conversion in only $10 \mathrm{~h}$ (see Figure S1 in the Supporting Information). Styrene, on the other hand, yielded a very low dispersity homopolymer in a well controlled fashion, as already well documented in the literature ${ }^{[27]}$ (SI, Figure S2).

For the copolymerization experiments, since the concentration of the two monomers could be monitored independently, the individual comonomer inclusion rate into the polymers during the reaction could be determined, confirming that SDPP is incorporated at a faster rate than styrene. All the experiments were stopped after ca. $80 \%$ global monomer conversion. For the copolymerizations using $\mathrm{f}_{\mathrm{SDPP}}=0.15$ and 0.25 , the comonomer consumption followed rather closely a pseudo $1 \mathrm{st}$ order rate law (Figure 2). Bending of the $1^{\text {st }}$ order plot beyond $70 \%$ conversion could be related to loss of a fraction of active chain ends, a phenomenon that is well documented for the ATRP of styrenic monomers at high conversions. ${ }^{[32]}$ In principle, a deviation from $1^{\text {st }}$ order behavior could also be caused by the reactivity ratios and the resulting comonomer ratio drift with concentration. This effect, however, should be too small to be clearly visible in this plot and should ultimately reflect into a small deviation of the phosphine comonomer distribution along the polymer chain (small concentration gradient). The copolymerization using $f_{\text {SDPP }}=0.5$ was faster, in addition of being less well controlled as judged from the dispersity of the recovered polymer (Table 1). The polymers recovered from the $f_{\text {SDPP }}=0.25$ and 0.15 copolymerizations show properties in agreement with a controlled radical polymerization process. The polymer molecular weight evolutions with conversion are close to linear, not significantly perturbed by small drift of comonomer ratio during polymerization (see Figure 3), and the dispersities are rather low. The corresponding data for the $\mathrm{f}_{\mathrm{SDPP}}=0.5$ copolymerization (see SI, Figure S3) shows a much less controlled behavior.

Since the composition is constantly changing, the refractive index of the polymer solutions is also in continuous evolution. Therefore, a MALLS detector was used to determine the copolymers molecular weight. The polymers $M_{n}$ are greater than those expected on the basis of the monomer/initiator ratio and conversion, corresponding initiator efficiencies in the $41-65 \%$ range. These initiator efficiencies are typical for this initiator and are generally attributed to side reactions that may occur for the primary radical when this is generated too rapidly relative to the rate of addition to monomer. ${ }^{[33-34]}$

The ${ }^{1} \mathrm{H}$ NMR analysis of the isolated polymers $\left(f_{\text {SDPP }}=0,0.15\right.$ and 0.25$)$ provided useful information on the chain ends. The region between $\delta 3$ and 5 clearly shows the chain end functions (the initiator $-\mathrm{OCH}_{2} \mathrm{CH}_{3}$ signal at ca. $\delta 3.6$ and the terminating $\mathrm{CHPhBr}$ signal at ca. $\delta 4.5)$, see Figure S5. The approximately correct 2:1 ratio of these two resonances allows the estimation that at least $90 \%$ of the halogen functionalities were preserved. Furthermore, the spectra reveal minor sharp resonances attributable to vinyl protons in the region between $\delta 5$ and 6 . Extensive efforts were made to remove all traces of residual styrene monomer from the isolated polymer, but it is not possible to exclude that these resonances are simply due to such impurity, especially given the narrow linewidth relative to the polymer resonances. On the other hand, they could also be attributed to the polymer unsaturated chain ends that are generated by dehydrobromination processes (Scheme 1). In that case, the ${ }^{1} \mathrm{H}$ NMR resonance integration combined with knowledge of the average degree of polymerization accounts for no more than $10 \%$ of such unsaturated chain ends.

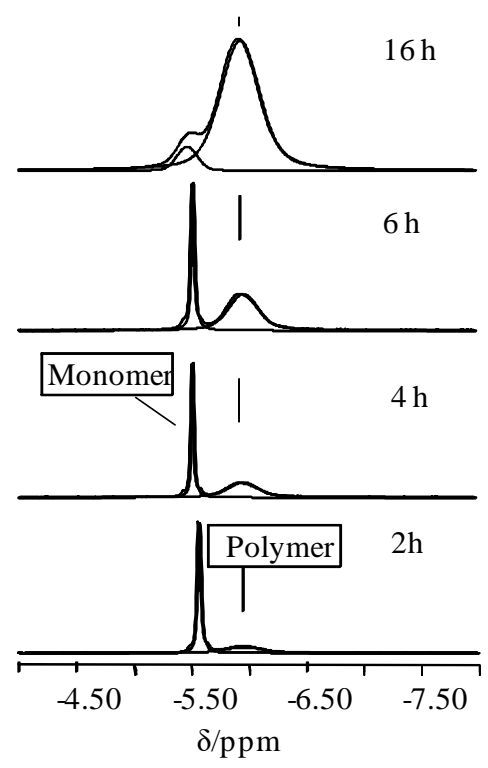

Figure 1. Evolution of the SDPP conversion for the copolymerization with $f_{S D P P}=0.25$, by ${ }^{31} \mathrm{P}$ NMR monitoring in $\mathrm{CDCl}_{3}$.

Further investigations were carried out for the copolymers at $\mathrm{f}_{\mathrm{SDPP}}=0.25$ and 0.15 . Adequate quantities of five different copolymers were prepared by quenching the polymerization reaction at different times. The characterization data of the purified polymers are summarized in

Once again, the polymerizations occurred in a controlled manner. The molecular weight distributions were uniform as shown by the analysis of selected SEC traces (see Figure 4). 


\begin{tabular}{|c|c|c|c|c|c|c|c|}
\hline$f_{S D P P}$ & $\mathrm{EBiB}{ }^{[\mathrm{b}]} / \mathrm{S} / \mathrm{SDPP} / \mathrm{Cu}^{[\mathrm{c}]} / \mathrm{L}$ & time $(\mathrm{h})$ & $\begin{array}{l}\text { conv. S } \\
(\%)\end{array}$ & $\begin{array}{l}\text { conv. SDPP } \\
(\%)\end{array}$ & $M_{n, \mathrm{th}}^{[\mathrm{d}]}$ & $M_{n, \mathrm{SEC}}$ & $\bigoplus$ \\
\hline 0 & $1: 50: 0: 0.5: 0.5$ & 20 & 81 & -- & 4420 & 6800 & 1.04 \\
\hline 0.15 & $1: 85: 15: 0.5: 0.5$ & 32 & 73 & 80 & 10120 & 18300 & 1.37 \\
\hline 0.25 & $1: 37.5: 12.5: 0.5: 0.5$ & 24 & 82 & 92 & 6710 & 12570 & 1.29 \\
\hline 0.5 & $1: 25: 25: 0.5: 0.5$ & 24 & 83 & 94 & 9130 & 22220 & 1.51 \\
\hline 1 & $1: 0: 50: 1: 1$ & 24 & -- & 97 & 13890 & 19680 & 1.36 \\
\hline
\end{tabular}
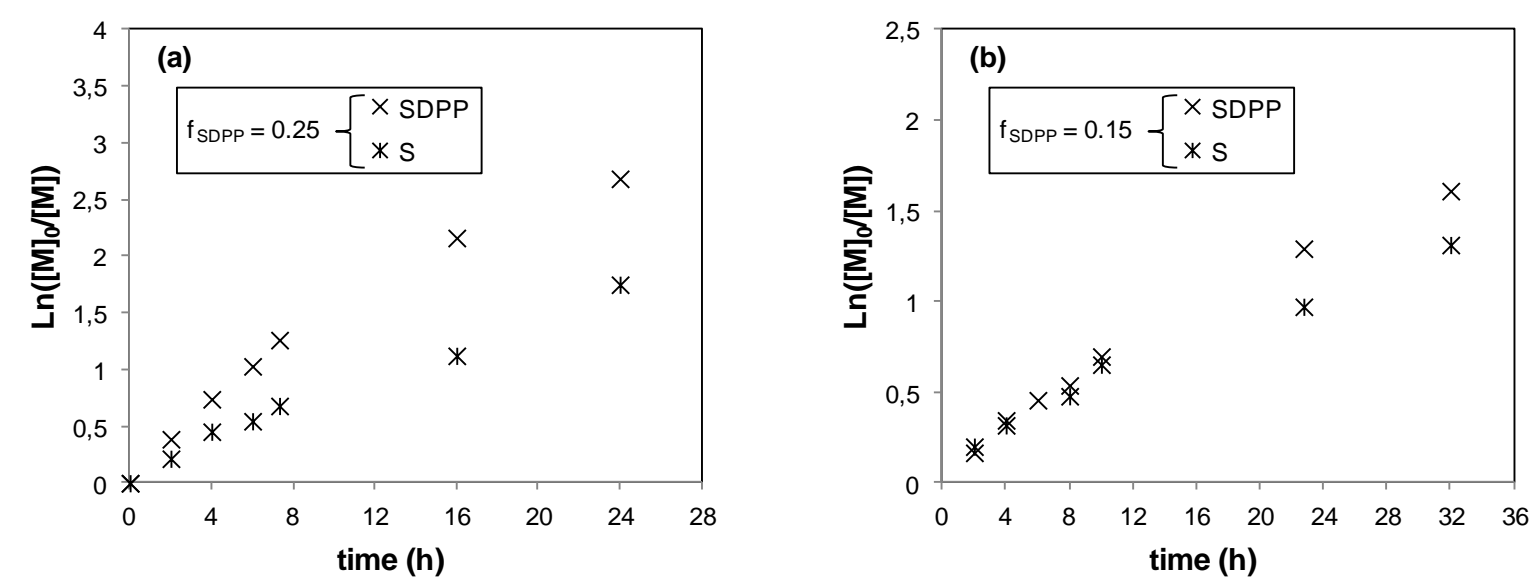

Figure 2. Semilogarithmic kinetic plots for the copolymerizations of SDPP and Styrene at $100^{\circ} \mathrm{C}$ in toluene: $f_{S D P P}=0.25(a)$ and $0.15(b) \cdot C o n d i t i o n s$ are given in Table 1.
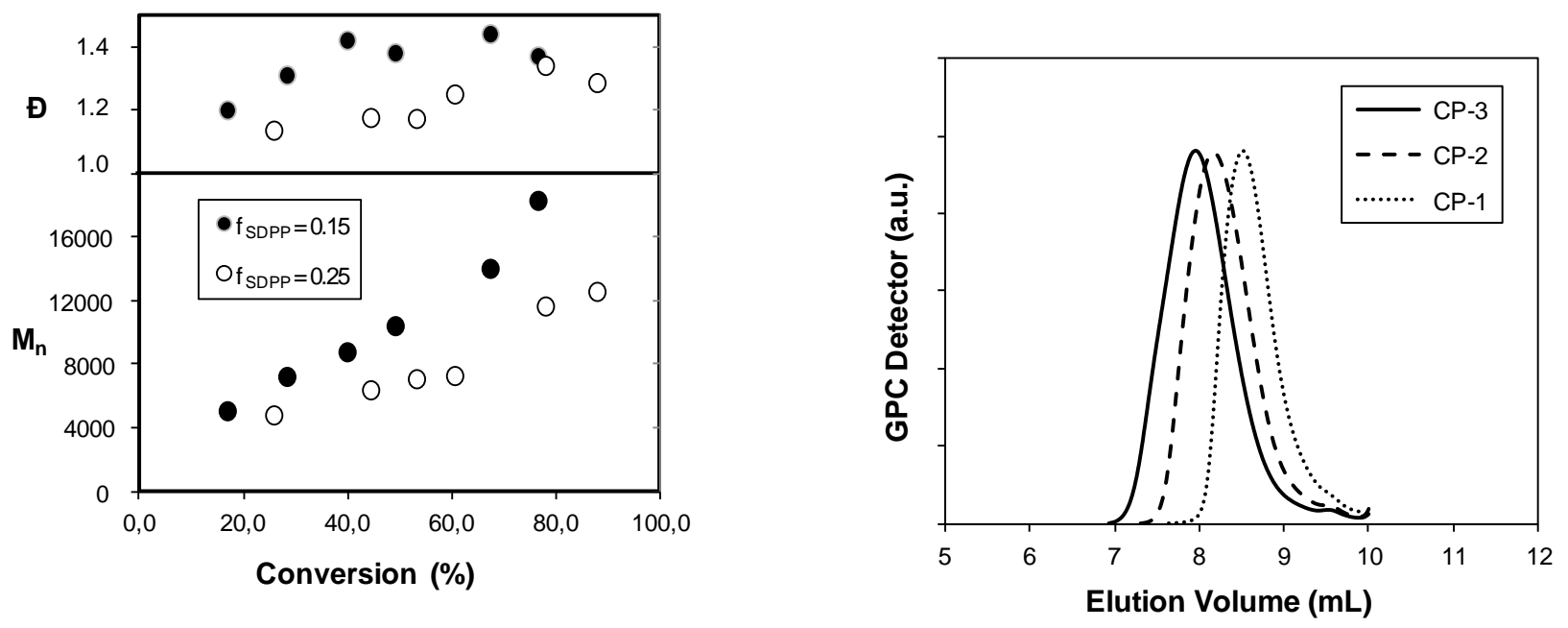

Figure 3. Evolution of the molecular weight and dispersity as a function of total monomer conversion for the copolymerization with $\mathrm{SSDPP}=0.25$ and 0.15 . 
Figure 4. Size exclusion chromatograms (RI detector) for the three isolated Poly(SDPP-s-Sty) obtained with fSDPP $=0.25$.

\begin{tabular}{|c|c|c|c|c|c|c|c|c|c|c|}
\hline \multirow{2}{*}{ Entry } & \multirow{2}{*}{$\begin{array}{l}f_{S D P P} \\
\text { initial feed }\end{array}$} & \multirow{2}{*}{ time $(\mathrm{h})$} & \multirow{2}{*}{ conv. S (\%) } & \multirow{2}{*}{ conv. SDPP (\%) } & \multicolumn{2}{|c|}{ 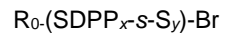 } & \multirow{2}{*}{$M_{n, \mathrm{SEC}}{ }^{[\mathrm{a}]}$} & \multirow{2}{*}{$\oslash$} & \multirow{2}{*}{$\mathrm{dn} / \mathrm{dc}$} & \multirow{2}{*}{$\begin{array}{l}\text { P content } \\
(\mathrm{mmol} / \mathrm{g})^{[b]}\end{array}$} \\
\hline & & & & & $x$ & $y$ & & & & \\
\hline CP-1 & 0.25 & 2.25 & 26 & 31 & 9 & 22 & 4900 & 1.19 & 0.181 & 1.77 \\
\hline CP-2 & 0.25 & 6 & 42 & 56 & 12 & 40 & 7520 & 1.38 & 0.210 & 1.54 \\
\hline CP-3 & 0.25 & 18 & 70 & 86 & 16 & 57 & 10270 & 1.33 & 0.200 & 1.48 \\
\hline CP-4 & 0.15 & 11.5 & 30 & 57 & 15 & 59 & 10490 & 1.38 & 0.212 & 1.40 \\
\hline CP-5 & 0.15 & 32 & 73 & 80 & 26 & 103 & 18300 & 1.37 & 0.204 & 1.41 \\
\hline
\end{tabular}

[a] From the MALLS detector. [b] Quantified from ${ }^{31} \mathrm{P}-\mathrm{NMR}$ analysis (refers only to the non oxidized phosphine). For reference, the ideal polymer with $25 \%$ incorporation of SDPP has a P content of $1.64 \mathrm{mmol} / \mathrm{g}$.

\section{(b) Application in 1-octene hydroformylation}

The copolymers obtained as described above were used as ligands for the Rh-catalyzed hydroformylation of octene under homogenous conditions. The desired reaction products are 1nonanal (linear isomer) and 2-methyl-1-octanal (branched isomer), with the main by-products being internal octene isomers derived from catalyzed isomerization, see Scheme 2. Other possible byproducts derive from hydrogenation (octane), hydroformylation products of the isomerized olefins, and products of further aldehyde hydrogenation (alcohols).

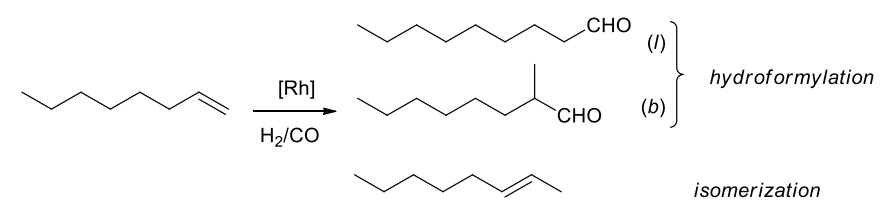

Scheme 2. Possible products from the catalyzed hydroformylation of 1-octene.

The key reaction parameters (solvent, temperature, catalyst concentration and $\mathrm{P} / \mathrm{Rh}$ ratio) have already been optimized for the homogeneous hydroformylation of 1-octene catalyzed by $\mathrm{PPh}_{3}$ and rhodium. ${ }^{[35-37]}$ Our choice of conditions for the catalytic tests was inspired by these previous investigations. We chose to work in toluene because this is a good solvent for the phosphinefunctionalized polymers described above. The active catalytic species is formed in situ by reaction of $\mathrm{Rh}(\mathrm{acac})(\mathrm{CO})_{2}$ and the phosphine ligand in the presence of $\mathrm{H}_{2} \cdot{ }^{[38-39]}$ The results are summarized in Table 3.

The polymers were used directly as isolated from the ATRP synthesis, therefore still contain the benzylic bromine atom as chain end as revealed by ${ }^{1} \mathrm{H}-\mathrm{NMR}$ (Figure S5). The reaction progress and the material balance were monitored by gas chromatography; the total amount of aldehydes produced was consistent with the syngas consumption. In order to verify whether the presence of the residual halogen end group perturbs the hydroformylation process, two additional experiments were carried out using $\mathrm{PPh}_{3}$ in the presence of 1-phenylethylbromide (run 2) or bromine-terminated polystyrene (run 3), in amounts chosen to emulate the $\mathrm{PPh}_{3} / \mathrm{Br}$ ratio of run 4. The conversion, initial rate and selectivity were very close to those found from the standard $\mathrm{PPh}_{3}$ system (run 1), suggesting the absence of interference of the benzylic bromide functions in hydroformylation under these conditions.

\begin{tabular}{|c|c|c|c|c|c|c|}
\hline Run & Ligand & $\mathrm{P} / \mathrm{Rh}$ & $\begin{array}{l}\text { time } \\
\text { (h) }\end{array}$ & $\begin{array}{l}\text { Residual } \\
\text { 1-octene }(\%)^{b}\end{array}$ & $\begin{array}{l}1 / b \\
\text { ratio }^{b}\end{array}$ & $\begin{array}{l}\text { (Init. rate) } \times 10^{4} \\
\left(\mathrm{~mol} \mathrm{l}^{-1} \mathrm{~s}^{-1}\right)^{c}\end{array}$ \\
\hline 1 & $\mathrm{PPh}_{3}$ & 8 & 0.33 & 7 & 2.7 & 28.5 \\
\hline 2 & $\mathrm{PPh}_{3}{ }^{d}$ & 8 & 0.33 & 10 & 2.8 & 25.0 \\
\hline 3 & $\mathrm{PPh}_{3}{ }^{e}$ & 8 & 0.33 & 9 & 2.5 & 29.1 \\
\hline 4 & CP-1 & 8 & 2 & 10 & 3.8 & 7.9 \\
\hline 5 & CP-2 & 8 & 2 & 17 & 4.7 & 4.2 \\
\hline 6 & CP-3 & 8 & 2 & 16 & 4.9 & 4.8 \\
\hline 7 & CP-3 & 4 & 2 & 12 & 3.8 & 8.7 \\
\hline 8 & CP-3 & 15 & 2 & 16 & 5.0 & 4.0 \\
\hline 9 & CP-4 & 8 & 2 & 15 & 4.4 & 5.9 \\
\hline 10 & CP-5 & 8 & 2 & 14 & 3.9 & 7.5 \\
\hline \multicolumn{7}{|c|}{$\begin{array}{l}\text { [a] }\left[\mathrm{Rh}(\mathrm{acac})(\mathrm{CO})_{2}\right]=2.0 \times 10^{-3} \mathrm{M} ;[1-\mathrm{octene}]=1.0 \mathrm{M} ; \mathrm{T}=90 \stackrel{\circ}{\circ} \mathrm{C} \text {; syngas } \\
\text { pressure }=20 \mathrm{bar}\left(\mathrm{CO} / \mathrm{H}_{2}=1\right) \text {. [b] From the GC data. } \mathrm{l} / \mathrm{b} \text { : linear to } \\
\text { branched aldehyde ratio. [c] Derived from the slope at the beginning of } \\
\text { the reaction of the number of moles produced vs time according to the } \\
\text { syngas consumption. [d] In the presence of PhCHBrCH}\left(2.0 \times 10^{-3} \mathrm{M}\right) \text {. } \\
\text { [e] In the presence of } \mathrm{PS}-\mathrm{Br}\left(\mathrm{M}_{\mathrm{n}}=3820 \mathrm{~g} \cdot \mathrm{mol}^{-1} ; 2.0 \times 10^{-3} \mathrm{M} \text { of } \mathrm{Br}\right. \\
\text { functions). }\end{array}$} \\
\hline
\end{tabular}

Runs 4-6 were carried out with the three polymers obtained using $\mathrm{f}_{\mathrm{SDPP}}=0.25(\mathrm{CP}-1, \mathrm{CP}-2$ and $\mathrm{CP}-3)$ under the same conditions and notably with the same $\mathrm{P} / \mathrm{Rh}$ ratio. Two additional experiments (runs 7 and 8 ) were carried out with CP-3 at different $\mathrm{P} / \mathrm{Rh}$ ratios. Finally, runs 9 and 10 show the results obtained with the two polymers prepared using $\mathrm{f}_{\mathrm{SDPP}}=0.15$. The reaction chemoselectivity was excellent since according to the GC/FID analyses aldehydes were the only detected products of the 
reaction except for a minor amount of isomerization: 1-nonanal (linear) and 2-methyl octanal (branched product) were the two major products. Neither other aldehyde isomers, nor octane or aldehyde hydrogenation products (alcohols) were detected. Two octene isomers were detected by GC/MS but in all cases they accounted for less of $1 \%$ of the converted 1 -octene. For the same system, Uenveren and Schomäcker ${ }^{[36]}$ observed a greater degree of isomerization at the initial stages of the reaction when using the $\mathrm{PPh}_{3}$ ligand. A rather interesting observation is that the linearto-branched $(1 / b)$ selectivity increases when switching from $\mathrm{PPh}_{3}$ to the polymer supported ligands. Comparison of runs 4-6 shows that the selectivity also increases slightly with the polymer chain length at $f_{\mathrm{SDPP}}=0.25$. At similar chain length, the $\mathrm{l} / \mathrm{b}$ ratios obtained with the polymers containing lower phosphorus content (CP-4 and CP-5) are slightly smaller. It is known that the $\mathrm{l} / \mathrm{b}$ ratio increases with the $\mathrm{P} / \mathrm{Rh}$ ratio until reaching a plateau; ${ }^{[35]}$ comparison of runs $6-8$ shows that a $P / R h$ ratio of 8 is sufficient to yield the maximum selectivity for the polymer catalytic system.

The initial hydroformylation rate in the presence of the polymer supported phosphine ligands is always lower than with $\mathrm{PPh}_{3}$. This rate seems somewhat correlated with the $1 / \mathrm{b}$ ratio, as it decreases when the selectivity increases. In accordance with what was observed with $\mathrm{PPh}_{3},{ }^{[35]}$ increasing the $\mathrm{P} / \mathrm{Rh}$ ratio from 4 to 15 (runs 6-8) diminishes the reaction rate by a factor of 2 .

A selectivity improvement, as well as the slowdown of the reaction rate, for polymer-immobilized phosphine ligands had already been reported for the hydroformylation of substrates such as 1-pentene, ${ }^{[40-41]} 1$-hexene, ${ }^{[22]} 1$-octene ${ }^{[42]}$ and 1-dodecene ${ }^{[43]}$. For instance, Hartley et al. reported a $1 / b$ ratio of 6 for the hydroformylation of 1 -hexene at $85^{\circ} \mathrm{C}$ using a phosphinated polypropylene, ${ }^{[22]}$ twice the value obtained for the monomeric catalyst. ${ }^{[4-45]}$ The decrease in the reaction rate is generally rationalized in terms of a reduced accessibility of the catalytic sites for the olefin; the immobilization and confinement of the complex into the polymer coils limits the diffusion rate of the catalytic complex and the olefin access inside the polymer, penalizing the reaction rate. However, our observation that the rate does not have a strong dependence on the polymer size or phosphine content shows that the olefin diffusion into the polymer folded chains is not a kinetically relevant process or that the difference between the chain lengths is too small to detect this effect. The Rh atoms may also play the role of intra-chain or interchain crosslinkers, leading to diffusional limitation. Because of the low polymer concentrations used in our catalytic experiments and the good solvent properties, the macromolecular chains are likely to be relatively unfolded and isolated from each other. Hence, intra-chain cross-linking should be dominant. Furthermore, for entropic reasons, coordination of the $\mathrm{Rh}$ atom by adjacent phosphine donors in the polymer chain should be preferred. Consequently, the macromolecule should remain flexible and the catalytic centers should continue to be relatively accessible. The relatively small number of SDPP units per chain (see Table 2) and the high $\mathrm{P} / \mathrm{Rh}$ ratio lead us to predict that the chains will only contain 1-3 Rh atoms on average (close to one for CP-1, two for CP-3 and CP-4, and 3 for CP-5). This low degree of crosslinking relative to the chain length, especially in a good solvent, may not be sufficient to influence the activity.

Following the extensive mechanistic studies of this catalyzed reaction, the catalyst structure for the $\mathrm{PPh}_{3}$ system is known to be of type $\mathrm{RhX}(\mathrm{CO})_{2}\left(\mathrm{PPh}_{3}\right)_{\mathrm{n}}$ or $\mathrm{RhX}(\mathrm{CO})\left(\mathrm{PPh}_{3}\right)_{\mathrm{n}}(\mathrm{X}=\mathrm{H}$, alkyl or acyl as the molecule moves around the catalytic cycle), containing between one and three phosphine ligands and yielding different catalytic performance. ${ }^{[46]}$ The lower activity of the polymersupported catalyst may be related to the chelating nature of the macromolecular ligand, disfavoring the more active and less regioselective configurations with a lower number of coordinated phosphine ligands. ${ }^{[35,47-49]}$ Their higher selectivity may also result from other polymer structuring effects. Two adjacent phosphines are separated by 3-4 styrene units on average for the CP-1, CP-2 and CP-3 systems, or 5-6 units for CP-4 and CP-5. These arrangements could have a direct influence on the spatial conformation of the substituents on the rhodium complex. It is known that the P-Rh-P bite angle is critical in terms of controlling the relative stability of equatorial-equatorial and equatorial-axial bis-phosphine isomers in the pentagonal bipyramidal geometry with strong repercussion on the $1 / \mathrm{b}$ selectivity, larger bite angles favoring the more selective eq.-eq. isomer. ${ }^{[50-51]}$ Hence, the greater selectivity may be induced by the imposition of a slightly greater (on average) bite angle by the constraints of the chain folds. For the polymers obtained from the $f_{S D P P}=0.25$ comonomer feed, the observed increase of the $\mathrm{l} / \mathrm{b}$ ratio with chain length may result from an increase of structural rigidity. On the other hand, the lower $1 / b$ ratio and relative insensitivity of this ratio as a function of chain length for the polymers resulting from the $\mathrm{f}_{\mathrm{SDPP}}=0.15$ comonomer feed could result from the longer average spacing between the phosphine ligands and therefore the greater flexibility of the polymer chain backbone, relaxing the structure towards thermodynamically more favored situations of lower bite angles. This situation is summarized in the schematic drawing of Figure 5.

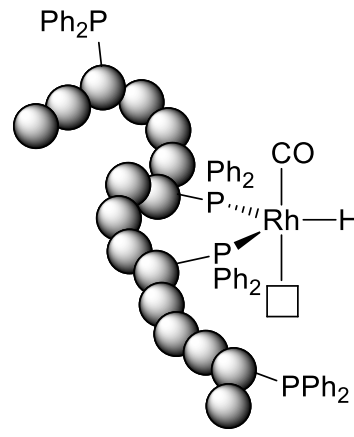

shorter P-P spacing

may favor eq-eq

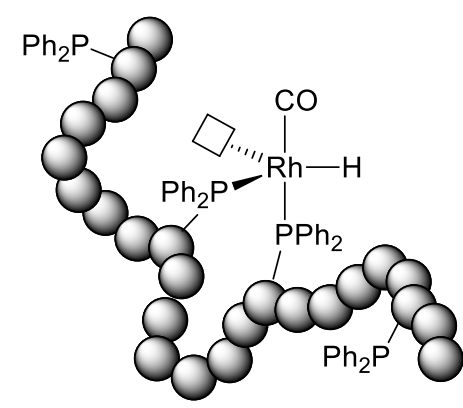

longer $P$-P spacing may favor eq-ax
Figure 5. Schematic drawing of bidentate coordination for the catalytically active pentagonal bipyramidal $\mathrm{Rh}$ center in the $\mathrm{HRh}(\mathrm{CO})\left(\mathrm{Polymer} \mathrm{PPh}_{3}\right)_{2}$ intermediate (the small square symbolizes the open coordination site).

\section{Conclusion}

In this contribution, we have described the first generation of triphenylphosphine ligands anchored on linear polystyrene chains by controlled radical polymerization. This method of synthesis allows easy tailoring in terms of polymer chain length - with a narrow molecular weight distribution - and phosphine incorporation. Application of these polymer supported phosphines in Rh-catalyzed 1-octene hydroformylation provides comparable results to those obtained with $\mathrm{PPh}_{3}$-containing polymers obtained by other less controlled methods in terms of activity and selectivity but allows for the first time an analysis of these parameters as a function of chain length and degree of phosphine 
incorporation. The study reveals a slight dependence of the hydroformylation linear/branched selectivity, for comparable phosphine incorporation, on the polymer chain length for chains containing ca. $25 \%$ of functionalized monomers. In perspective, this synthetic method allows a better control of the polymer architecture and thereby a finer tailoring of the ligand performance. It also allows taking advantage of the residual brominated chain end to elaborate more complex polymer architectures for more specific catalytic applications. Studies in this direction are currently ongoing in our laboratories.

\section{Experimental Section}

Materials. Unless otherwise stated, all operations were carried out under an argon atmosphere. Styrene $(99 \%$, Aldrich) was distilled under reduced pressure from $\mathrm{CaH}_{2}(90-95 \%$, Aldrich) and stored under argon at $-20^{\circ} \mathrm{C}$ before use. Toluene $(99.7 \%$, Aldrich) was dried and distilled under argon. $\mathrm{CuBr}$ (99.999\%, Aldrich), $\mathrm{CuBr}_{2}$ (99.0\%, Fluka), triphenylphosphine (99\%, Aldrich), $\mathrm{Rh}(\mathrm{CO})_{2}(\mathrm{acac})(99 \%$ Alfa Aesar), hexane $(>96.5 \%$, Aldrich), anisole $(99.0 \%$, Fluka), octane (>99.0\%, Aldrich), 1-octene (99+\%, ACROS), nonanal (>97\%, Alfa Aesar), dodecane (99\%, Aldrich), ethyl 2-bromoisobutyrate EBiB (98\%, Aldrich), 1-phenyl ethylbromide 1-PEB (97\%, Aldrich), ethyl diphenyl phosphine oxide (98\%, Lancaster) and SDPP (97\%, Aldrich) were used without further purification. Tris(2(dimethylamino)ethyl)amine ( $\mathrm{Me}_{6}$ TREN) was synthesized according to a literature protocol. ${ }^{[27]}$ Carbon monoxide and hydrogen were supplied by Linde gas S.A. Syngas was prepared by mixing equivalent molar amounts of $\mathrm{H}_{2}$ and $\mathrm{CO}$ in a reservoir vessel.

Instrumentation. The conversion during the SDPP polymerization was monitored and the phosphorus content in the purified polymers was measured by ${ }^{31} \mathrm{P}\left\{{ }^{1} \mathrm{H}\right\}$ NMR with a Bruker Avance 300 spectrometer equipped with a $5 \mathrm{~mm}$ TXO probe head. Chemical shifts are reported in ppm relative to tetramethylsilane $\left({ }^{1} \mathrm{H}\right)$, referenced to the chemical shift of residual solvents resonances, or from external $85 \% \mathrm{H}_{3} \mathrm{PO}_{4}\left({ }^{31} \mathrm{P}\right)$. All experiments were carried out in $\mathrm{CDCl}_{3}$ at 298.0 K. Long relaxation delays $(40 \mathrm{~s})$ were used for the quantitative $P$ analyses to insure full magnetization recovery, and the relative peak area was calculated by signal deconvolution and fitting procedures. Absolute amounts were determined relative to known added amounts of ethyl diphenylphosphine oxide (EtDPPOx) as an internal standard. The phosphine content was determined directly from their relative intensities from the ${ }^{31} \mathrm{P}\left\{{ }^{1} \mathrm{H}\right\}$ NMR spectra:

$$
P \text { content }=\frac{m_{r e f}}{M w_{r e f}} * \frac{I_{\text {pol }}}{I_{r e f} * m_{p o l}} \quad\left[\frac{\text { mol } P}{g_{\text {polymer }}}\right]
$$

where $M w_{\text {ref }}$ is the molecular weight of EtDPPOx, $m_{\text {ref }}$ and $m_{p o l}$ are the weights of EtDPPOx and polymer respectively. $I_{\text {ref }}$ and $I_{\text {pol }}$ are the intensities from the spectra. Size exclusion chromatographic analyses (SEC) were carried out on a PL gel $(5 \mu \mathrm{m}$ particles, $50 \times 7.5 \mathrm{~mm})$ guard column and a PL-gel $5 \mu \mathrm{m}$ mixed-D $(300 \times 7.5 \mathrm{~mm})$ column (Polymer Laboratories), in filtered THF as eluent. Molecular weights were measured by a multiangle light-scattering (MALLS) detector (minidawn Tristar, Wyatt Technology Corporation) coupled with a refractive index detector (RI2000, Sopares), set at $35^{\circ} \mathrm{C}$. Molecular weight values for the copolymers and the SDPP homopolymer were determined using the MALLS detector. The total mass recovery protocol was employed, which in turn yields the corresponding $\mathrm{dn} / \mathrm{dc}$ values. On the other hand, a literature value of $d n / d c$ was used for polystyrene (0.184). Gas chromatographic analyses were carried out on a Trace GC 2000 Thermo Fisher chromatograph equipped with a flame ionization detector (FID). The separations were carried out with a capillary CP-WAX 52 CB $(25 \mathrm{~m} \times 0.25 \mathrm{~mm}, 0.2 \mu \mathrm{m}$ film thickness $)$ column using helium as carrier gas. In order to follow the styrene conversion during the polymerizations, dodecane was used as an internal standard. For the hydroformylation reactions, samples withdrawn for kinetic monitoring were diluted into diethyl ether and anisole was added as internal standard. Prior to the analytical tests, standard solutions were prepared in the range of investigated concentrations of reactant (1-octene) and product ( $n$-nonanal), and calibration curves were plotted for quantification. It was assumed that the response factor is the same for isomers as for the linear compounds. The identification of the compounds was confirmed by GC/MS analysis.

Synthetic protocols. All the polymerizations were performed in glass flasks equipped with a three way stopcock and a magnetic stir bar. Solvents were flashed by an argon flow and introduced using argonpurged syringes fitted with stainless steel needles. For the homopolymerization of styrene, the polymer purification was carried out in air.

Styrene homopolymerization. In a typical experiment, $\mathrm{CuBr}(55.3$ $\mathrm{mg}, 0.38 \mathrm{mmol}), \mathrm{CuBr}_{2}(9.4 \mathrm{mg}, 0.04 \mathrm{mmol})$, toluene $(13 \mathrm{~mL})$, $\mathrm{Me}_{6}$ TREN (110 $\left.\mu \mathrm{L}, 0.42 \mathrm{mmol}\right)$, styrene $(5.0 \mathrm{~mL}, 42 \mathrm{mmol})$ and dodecane $(1 \mathrm{~mL})$ were sequentially introduced in a $50 \mathrm{~mL}$ roundbottomed Schlenk flask at room temperature. EBiB $(128 \mu \mathrm{L}, 0.84$ $\mathrm{mmol}$ ) was then introduced with a microsyringe and the mixture was rapidly cooled-down by immersion into a liquid nitrogen-filled Dewar flask. The mixture was degassed by three freeze-pump-thaw cycles. The flask was then immersed in an oil bath thermostated at $100^{\circ} \mathrm{C}$ to start the reaction. At timed intervals, aliquots of the reaction solution were withdrawn with argon purged syringes, followed by filtration through a small neutral-alumina column in order to remove the catalyst. One part of the colorless samples was analyzed by gas chromatography to determine the conversion and the remaining part was used for the SEC analysis. After 20 hours, the Schlenk flask was rapidly cooled down and the mixture was diluted with toluene and then filtered through a neutral-alumina column. The resulting solution was concentrated by evaporation under reduced pressure and the polymer was precipitated by addition of cold hexane, filtered off and dried under vacuum until total residual solvent evaporation (ca. $3 \mathrm{~d}$ ) to yield a white powder. Yield $3.6 \mathrm{~g}(82 \%) . M_{n, \mathrm{SEC}}=6800 \mathrm{~g} / \mathrm{mol} ; \oplus=1.04$.

Stability test of $\mathrm{PPh}_{3}$ under ATRP conditions: phosphonium salt formation. $\mathrm{CuBr}(26.3 \mathrm{mg}, 0.18 \mathrm{mmol}), \mathrm{CuBr}_{2}(4.5 \mathrm{mg}, 0.02 \mathrm{mmol})$ and $\mathrm{PPh}_{3}(1.311 \mathrm{~g}, 5 \mathrm{mmol})$ were charged on a round-bottom flask with a magnetic stir bar. The flask was degassed and filled with argon by three vacuum-argon cycles. Toluene $(5.0 \mathrm{~mL})$ and $\mathrm{Me}_{6}$ TREN (55 $\mu \mathrm{L}, 0.2 \mathrm{mmol}$ ) were added in turn using argon-purged syringes. Then, 1-phenylethyl bromide ( $55 \mu \mathrm{L}, 0.4 \mathrm{mmol}$ ) was introduced with a microsyringe and the mixture was rapidly cooled-down by immersion into a liquid nitrogen-filled Dewar flask. The mixture was degassed by three freeze-pump-tawl cycles to remove oxygen traces. The flask was then immersed in an oil bath thermostated at $100^{\circ} \mathrm{C}$ for $26 \mathrm{~h}$. At timed intervals, aliquots of the reaction solution were withdrawn via argon purged syringes, followed by filtration under argon through a small neutral-alumina column in order to remove the metal complexes, and finally analyzed by ${ }^{31} \mathrm{P}$ NMR spectroscopy.

SDPP-S copolymerization. The same procedure was used to obtain an SDPP homopolymer or an SDPP/S statistical copolymer. Only the reaction carried out with $\mathrm{f}_{\mathrm{SDPP}}=0.25$ will be described in detail: in a 50 $\mathrm{mL}$ round-bottom Schlenk flask were sequentially introduced $\mathrm{CuBr}$ (59.3 mg, $0.41 \mathrm{mmol}), \mathrm{CuBr}_{2}(10.1 \mathrm{mg}, 0.05 \mathrm{mmol}), \operatorname{SDPP}(3.344 \mathrm{~g}$, $11.25 \mathrm{mmol}$ ), toluene $(15 \mathrm{~mL}), \mathrm{Me}_{6} \operatorname{TREN}(120 \mu \mathrm{L}, 0.45 \mathrm{mmol})$, styrene $(4.0 \mathrm{~mL}, 33.75 \mathrm{mmol})$ and dodecane $(1 \mathrm{~mL})$ at room temperature. Then EBiB $(140 \mu \mathrm{L}, 0.9 \mathrm{mmol})$ was introduced with a microsyringe and the mixture was rapidly cooled by immersion into a liquid-nitrogen-filled Dewar flask. The solution was then degassed by three freeze-pump-thaw cycles and the flask was finally immersed in an oil bath thermostated at $100^{\circ} \mathrm{C}$. At timed intervals, aliquots of the reaction solution were withdrawn with argon purged syringes, followed by filtration under argon through a small neutral-alumina column in order to remove the catalyst. One part of the colorless samples was analyzed by gas chromatography to determine the styrene conversion and the remaining part was used for the SEC and 
${ }^{31} \mathrm{P}$ NMR analyses. After 24 hours, the Schlenk flask was rapidly cooled down and the mixture was diluted with toluene and filtered through an neutral alumina column. The polymer was precipitated by addition of cold hexane $(0.5 \mathrm{~L})$, filtered off and dried under vacuum until total residual solvent evaporation (ca. $3 \mathrm{~d}$ ) to yield a white powder. Yield $4.2 \mathrm{~g}(60 \%) . M_{n, \mathrm{SEC}}=12570 \mathrm{~g} / \mathrm{mol} ; \emptyset=1.29$. The same reaction was repeated under the same conditions and quenched at different times in order to produce copolymer chains with different lengths: $\mathrm{CP} 1$ : reaction time $=2 \mathrm{~h} 15^{\prime}, M_{n, \mathrm{SEC}}=4900 \mathrm{~g} / \mathrm{mol} ; \emptyset=1.19$; $\mathrm{dn} / \mathrm{dc}=0.181 ; \mathrm{CP} 2$ : reaction time $=6 \mathrm{~h}, M_{n, \mathrm{SEC}}=7520 \mathrm{~g} / \mathrm{mol} ; \oplus$ $=1.38 ; \mathrm{dn} / \mathrm{dc}=0.210 ; \mathrm{CP} 3$ : reaction time $=18 \mathrm{~h}, M_{n, \mathrm{SEC}}=10270$ $\mathrm{g} / \mathrm{mol} ; \bigoplus=1.33 ; \mathrm{dn} / \mathrm{dc}=0.200 ; \mathrm{CP} 4$ : reaction time $=11 \mathrm{~h} 30$ ', $M_{n, \mathrm{SEC}}=$ $10490 \mathrm{~g} / \mathrm{mol} ; \emptyset=1.376 ; \mathrm{dn} / \mathrm{dc}=0.212 ;$ CP 5: reaction time $=32 \mathrm{~h}$, $M_{n, \mathrm{SEC}}=18300 \mathrm{~g} / \mathrm{mol} ; \emptyset=1.371 ; \mathrm{dn} / \mathrm{dc}=0.204$.

Homogeneous hydroformylation of 1-octene. Hydroformylation experiments were carried out in an autoclave reactor equipped with a gas inducing stirrer. The catalyst precursor $\mathrm{Rh}(\mathrm{CO})_{2}(\mathrm{acac})(53.2 \mathrm{mg}$, $0.2 \mathrm{mmol})$ and the desired amount of ligand $\left(\mathrm{PPh}_{3}\right.$ or the phosphinefunctionalized polymers CP 1-5, vide infra) were introduced into a Schlenk flask and degassed by three vacuum/nitrogen inletting cycles Then, a nitrogen-bubbled mixture of toluene $(84 \mathrm{~mL}), 1$-octene $(16 \mathrm{~mL}$ $102 \mathrm{mmol}$ ) and dodecane $(2 \mathrm{~mL})$ was introduced via cannula transfer. The resulting homogeneous system was stirred for 30 minutes and then transferred to the reactor vessel where a first sample was withdrawn. The system was purged with syngas to remove the remaining nitrogen. The reactor was then heated under a syngas atmosphere (2 bar) and at low stirring speed (140 r.p.m.) in order to hinder gas-liquid mass transfer and therefore limit the reaction extent. The catalyst complex is supposed to be formed in situ. When the desired reaction temperature was achieved (90 $\mathrm{C}$ ), stirring was stopped and syngas was introduced from the ballast into the reactor up to the desired pressure (20 bar). A sample was withdrawn to evaluate the amount of products eventually formed during the heating period. Then the reaction was initiated by increasing the agitation speed to $1200 \mathrm{rpm}$ and data acquisition was started. It was checked that this stirring speed is high enough for the reaction to occur in the chemical regime, as no improvement was found when increasing it to $1500 \mathrm{rpm}$. The initial hydroformylation rate was obtained from the pressure variations in the reservoir, feeding the reactor which was maintained at constant pressure. The selectivity toward linear aldehyde and the conversion were monitored by gas chromatographic analyses of additional liquid samples (after 20, 40 and 60 minutes for $\mathrm{PPh}_{3}$ and 30, 60, 90 and 120 minutes for the polymeric ligands) during the reaction. The amounts of polymeric ligand used, calculated on the basis of their phosphorus content, were: CP-1: $920 \mathrm{mg}$; CP-2: $1061 \mathrm{mg}$; CP-3: 549; 1098 and $2060 \mathrm{mg}$ for the P/Rh ratios of 4,8 and 15 respectively; CP-4: $1165 \mathrm{mg}$ and CP-5: $1160 \mathrm{mg}$. Two additional runs were done in which $\mathrm{PPh}_{3}(432 \mathrm{mg}, 1.63 \mathrm{mmol}$ ) was used as ligand in the presence of 1-phenylethyl bromide $(28 \mu \mathrm{L}, 0.2$ $\mathrm{mmol})$ or bromide-end polystyrene $\left(\mathrm{M}_{\mathrm{n}, \mathrm{SEC}}=3820 \mathrm{~g} / \mathrm{mol} ; 771.5 \mathrm{mg}\right.$, $0.2 \mathrm{mmol})$. The procedure was the same as described above.

\section{Acknowledgements}

We are grateful to the CNRS (Centre National de la Recherche Scientifique), the ANR (Agence Nationale de la Recherche, Grant No. ANR-11-BS07-025-01), and the IUF (Institut Universitaire de France) for financial support. We also thank the PRES University of Toulouse for a Ph.D. fellowship to A. F. Cardozo.

Keywords: Polymer supported phosphine ligands • Polymeric catalyst • Rhodium hydroformylation catalysis - ATRP • Anchored triphenylphosphine
[1] K. Matyjaszewski, Y. Gnanou, L. Leibler, Macromolecular Engineering: Precise Synthesis, Materials Properties, Applications, Wiley-VCH Verlag $\mathrm{GmbH}, 2007$.

[2] C. J. Hawker, A. W. Bosman, E. Harth, Chem. Rev. 2001, 101, 3661 3688.

[3] W. A. Braunecker, K. Matyjaszewski, Progr. Polym. Sci. 2007, 32, 93146.

[4] M. Ouchi, T. Terashima, M. Sawamoto, Chem. Rev. 2009, 109, 49635050

[5] F. di Lena, K. Matyjaszewski, Progr. Polym. Sci. 2010, 35, 959-1021.

[6] M. Destarac, Macromol. React. Engin. 2010, 4, 165-179.

[7] N. E. Leadbeater, M. Marco, Chem. Rev. 2002, 102, 3217-3273.

[8] M. Guino, K. K. Hii, Chem. Soc. Rev. 2007, 36, 608-617.

[9] J. Manassen, Chim. Ind. (Milan) 1969, 51, 1058-1062.

[10] R. H. Grubbs, L. C. Kroll, J. Am. Chem. Soc. 1971, 93, 3062-3063.

[11] R. H. Grubbs, L. C. Kroll, E. M. Sweet, J. Macromol. Sci. A 1973, A 7, 1047-1063.

[12] M. Capka, P. Svoboda, M. Cerny, J. Hetflejs, Tetrahedron Lett. 1971, 4787-4790.

[13] G. O. Evans, C. U. Pittman, R. McMillan, R. T. Beach, R. Jones, J. Organomet. Chem. 1974, 67, 295-314.

[14] D. C. Bailey, S. H. Langer, Chem. Rev. 1981, 81, 109-148.

[15] G. Challa, J. Reedijk, P. vanLeeuwen, Polym. Advan. Technol. 1996, 7, 625-633.

[16] C. A. McNamara, M. J. Dixon, M. Bradley, Chem. Rev. 2002, 102, 32753299.

[17] K. G. Allum, R. D. Hancock, I. V. Howell, R. C. Pitkethly, P. J. Robinson, J. Organomet. Chem. 1975, 87, 189-201.

[18] N. A. Demunck, M. W. Verbruggen, J. J. F. Scholten, J. Mol. Catal. 1981, 10, 313-330.

[19] R. Rabinowitz, R. Marcus, J. Pellon, J. Polym. Sci., Polym. Chem. 1964, 2, 1241-1249.

[20] M. Kwok, W. Choi, H. S. He, P. H. Toy, J. Org. Chem. 2003, 68, 9831 9834.

[21] H. G. Borner, W. Heitz, Macromol. Chem. Phys. 2000, 201, 740-746.

[22] F. R. Hartley, S. G. Murray, P. N. Nicholson, J. Mol. Catal. 1982, 16, 363-383.

[23] F. Shibahara, K. Nozaki, T. Matsuo, T. Hiyama, Bioorg. Med. Chem. Lett. 2002, 12, 1825-1827.

[24] P. W. N. M. van Leeuwen, T. Jongsma, G. Challa, Macromol. Symp. 1994, 80, 241-256.

[25] T. Terashima, M. Ouchi, T. Ando, M. Kamigaito, M. Sawamoto, J. Polym. Sci., Polym. Chem. 2006, 44, 4966-4980.

[26] T. Terashima, M. Ouchi, T. Ando, M. Kamigaito, M. Sawamoto, Macromolecules 2007, 40, 3581-3588.

[27] J. Queffelec, S. G. Gaynor, K. Matyjaszewski, Macromolecules 2000, 33 , 8629-8639.

[28] V. Coessens, K. Matyjaszewski, J. Macromol. Sci. A 1999, A36, 653-666.

[29] K. Matyjaszewski, J. H. Xia, Chem. Rev. 2001, 101, 2921-2990.

[30] J. P. A. Heuts, T. P. Davis, Macromol. Rapid Comm. 1998, 19, 371-375

[31] S. V. Arehart, K. Matyjaszewski, Macromolecules 1999, 32, 2221-2231.

[32] J. F. Lutz, K. Matyjaszewski, J. Polym. Sci., Polym. Chem. 2005, 43, 897-910.

[33] K. Matyjaszewski, J. L. Wang, T. Grimaud, D. A. Shipp, Macromolecules 1998, 31, 1527-1534.

[34] A. Mittal, S. Sivaram, J. Polym. Sci., Polym. Chem. 2005, 43, 4996-5008.

[35] R. M. Deshpande, B. M. Bhanage, S. S. Divekar, R. V. Chaudhari, J. Mol. Catal. 1993, 78, L37-L40.

[36] H. H. Y. Uenveren, R. Schomaecker, Catal. Lett. 2006, 110, 195-201.

[37] R. L. Pruett, J. A. Smith, Ger. Pat. 2,058,814 1971

[38] C. K. Brown, G. Wilkinson, J. Chem. Soc. A 1970, 2753-2764.

[39] E. Kwaskowskachec, A. M. Trzeciak, J. J. Ziolkowski, React. Kinet. Catal. Lett. 1984, 26, 21-24.

[40] C. U. Pittman, R. M. Hanes, Ann. New York Acad. Sci. 1974, 239, 76-87.

[41] C. U. Pittman, R. M. Hanes, J. Am. Chem. Soc. 1976, 98, 5402-5405.

[42] P. Kalck, E. L. de Oliveira, R. Queau, B. Peyrille, J. Molinier, J. Organomet. Chem. 1992, 433, C4-C8.

[43] N. S. Imyanitov, V. A. Rybakov, S. B. Tupitsyn, Petrol. Chem. 1992, 32 408-412.

[44] B. E. Hanson, M. E. Davis, Journal of Chemical Education 1987, 64 928-930. 
[45] R. M. Deshpande, R. V. Chaudhari, Ind. Engin. Chem. Res. 1988, 27, 1996-2002.

[46] P. W. N. M. van Leeuwen, C. Claver, in Catalysis by Metal Complexes, Vol. 22, Kluwer, Dordrecht, 2000.

[47] F. H. Jardine, Polyhedron 1982, 1, 569-605.

[48] R. L. Pruett, J. A. Smith, J. Org. Chem. 1969, 34, 327-330.

[49] D. Evans, J. A. Osborn, G. Wilkinson, J. Chem. Soc. A 1968, 3133-3142.

[50] Z. Freixa, P. W. N. M. van Leeuwen, Dalton 2003, 1890-1901.
[51] P. W. N. M. van Leeuwen, Homogeneous Catalysis. Understanding the Art, Kluwer Academic Publishers, Dordrecht, The Netherlands, 2004.

Received: ((will be filled in by the editorial staff))

Published online: ((will be filled in by the editorial staff) 


\section{Entry for the Table of Contents}

\section{FULL PAPER}

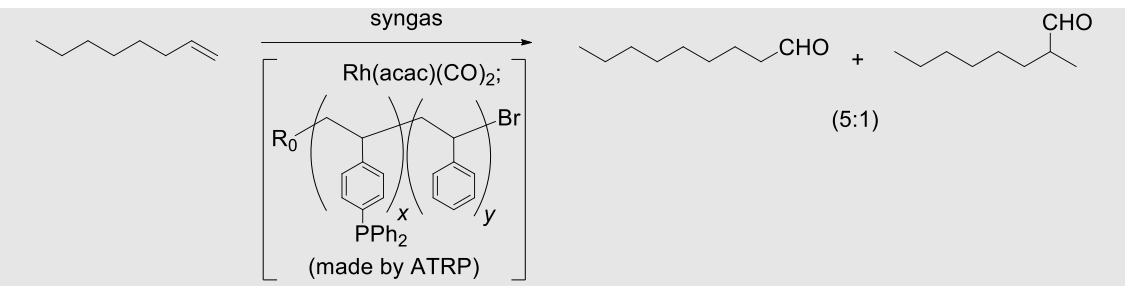

Controlled length polystyrene-supported $\mathrm{PPh}_{3}$ ligands were made for the first time by atom transfer copolymerization of styrene and styryldiphenylphosphine. Controlled $M_{n}$ and low dispersity are obtained for low SDPP content ( $\leq 25 \%)$.
The performance of these polymeric ligands in rhodium-catalyzed hydroformylation of 1-octene has been evaluated as a function of polymer length, degree of $\mathrm{P}$ incorporation and $\mathrm{P} / \mathrm{Rh}$ ratio.
Andrés F. Cardozo ${ }^{[a],[b],[c]}$, Eric

Manoury[a],[c], Carine Julcour $r^{[b],[c]}$, JeanFrançois Blanco ${ }^{[b],[c]}$, Henri Delmas ${ }^{[b],[c]}$, Florence Gayet, ${ }^{[a],[c]}$ Rinaldo Poli*

Page No. - Page No.

Preparation of Polymer Supported Phosphine Ligands by Metal Catalyzed Living Radical Copolymerization and Their Application to Hydroformylation Catalysis 